\title{
A NEW PROOF OF A THEOREM OF DENJOY, YOUNG, AND SAKS*
}

\author{
BY E. H. HANSON
}

In 1915 Denjoyt published the following theorem.

THEOREM. If $f(x)$ is any continuous function, then, at every point $x$ with the possible exception of a set of measure zero, either (1) $f(x)$ has a unique finite derivative; or (2) the upper (Dini) derivative on one side is $+\infty$, the lower derivative on the other side is $-\infty$, and the other two extreme derivatives are finite and equal; or (3) the two upper derivatives are $+\infty$ and the two lower derivatives are $-\infty$.

This result was extended to measurable functions by G. C. Young and to unrestricted functions by S. Saks. $\ddagger$ The basis for the latter proof is the corresponding theorem for monotone functions, that is, every monotone function has a unique finite derivative almost everywhere. § Another proof, as yet unpublished, is due to $\mathrm{H}$. Blumberg, who uses a general principle for passing from measurable functions to general functions.

The present paper gives a new proof of this theorem, for the general case, in a manner which lays bare the essential simplicity of the necessary reasoning involved. This proof, indeed, is a direct remodeling, by means of a few well known devices that may now be regarded as standardized in analysis, of the intuitive perception of the theorem which readily suggests itself. It appears, too, that there is no real gain in arguing with a special hypothesis such as continuity or measurability.

* Presented to the Society, December 2, 1933. The author wishes to express his appreciation to $\mathrm{H}$. Blumberg for helpful criticism.

$\dagger$ Journal de Mathématiques, (7), vol. 1, pp. 105-240.

$\ddagger$ G. C. Young, Proceedings of the London Mathematical Society, (2), vol. 15 (1916), pp. 360-384; S. Saks, Fundamenta Mathematicae, vol. 5 (1924), pp. 98-104. See also S. Banach, Comptes Rendus, vol. 173 (1921), pp. 457-459; A. S. Besicovitch, Akademiia Nauk, (6), vol. 19 (1925), pp. 97-122 and 527540 ; J. C. Burkill and U. S. Haslam-Jones, Proceedings of the London Mathematical Society, (2), vol. 32 (1931), pp. 346-355, and Quarterly Journal of Mathematics, (Oxford), vol. 4 (1933), pp. 233-239.

$\S$ Due to H. Lebesgue, Leçons sur l'Intégration, 1904, p. 128. 
It will be sufficient to prove that an arbitrary function $f(x)$ has almost everywhere either $D^{+} f(x)$ and $D_{-} f(x)$ finite and equal, or $D^{+} f(x)=+\infty, D_{-} f(x)=-\infty$. For, since $D^{-} f(x)=-D_{-}[-f(x)]$ and $D_{+} f(x)=-D^{+}[-f(x)]$, a similar statement must hold for $D^{-} f(x)$ and $D_{+} f(x)$ and the theorem follows.

We shall consider first the set of points at which $D^{+}$and $D_{-}$ are not both finite. We begin by showing that the set where $D^{+}=+\infty$ and $D_{-} \neq-\infty$ is of measure zero. Denote this set by $E$. Classifying, we have

$$
E=\sum_{r} E_{r}
$$

where $E_{r}$ is the set of points of $E$ at which $D_{-}>r$, and the summation is over all rational values of $r$. Furthermore

$$
E_{r}=\sum_{n=1}^{\infty} E_{r}^{(n)}
$$

where $E_{r}(n)$ is the set of points $x$ of $E_{r}$ at which $f(x)-f\left(x^{\prime}\right)$ $>r\left(x-x^{\prime}\right)$ for all $x^{\prime}$ between $x-1 / n$ and $x$. It will suffice to show that, for every pair $(r, n), E_{r}(n)$ is of measure zero. Let $(a, b)$ be any interval of length less than $1 / n$ with right end point $b$ in $E_{r}{ }^{(n)}$ and denote by $S$ the set of points of $E_{r}{ }^{(n)}$ interior to $(a, b)$. Let $k$ be any number whatsoever and consider the set $T$ of all intervals $\left(x, x^{\prime}\right)$ interior to $(a, b)$ for which the left end point $x$ is in $S$ and $f\left(x^{\prime}\right)-f(x)>k\left(x^{\prime}-x\right)$. Every point $x$ of $S$ is the left end point of an arbitrarily small interval of $T$. Hence, according to the Vitali Covering Theorem, ${ }^{*}$ there exist, for every $\epsilon>0$, a finite number of non-overlapping intervals of $T$, say $I_{\nu}=\left(x_{\nu}, x_{\nu}^{\prime}\right),(\nu=1,2, \cdots, p)$, such that

$$
\left|m_{e}(S)-\sum_{\nu=1}^{p} m\left(I_{\nu}\right)\right|<\epsilon,
$$

where $m_{e}$ stands for exterior measure and $m$ for measure. Furthermore, if $(\alpha, \beta)$ is one of the intervals complementary to the $I_{\nu}$ with respect to $(a, b), \beta$ is a point of $E_{r}^{(n)}$ and $\beta-\alpha<1 / n$. Hence $f(\beta)-f(\alpha)>r(\beta-\alpha)$. Therefore

$$
f(b)-f(a)>k \sum_{\nu=1}^{p} m\left(I_{\nu}\right)+r\left[b-a-\sum_{\nu=1}^{p} m\left(I_{\nu}\right)\right] .
$$

* Use is here made of a slight modification of the form in which the Vitali Theorem is customarily stated in that the points covered are allowed to be end points of the covering intervals. 
Substituting for $\sum_{\nu=1}^{p} m\left(I_{\nu}\right)$ its equivalent in terms of $m_{e}(S)$, we obtain

$$
f(b)-f(a)>k\left[m_{e}(S)+\epsilon^{\prime}\right]+r\left[b-a-m_{e}(S)-\epsilon^{\prime}\right],
$$

where $\left|\epsilon^{\prime}\right|<\epsilon$. It follows that

$$
f(b)-f(a) \geqq k m_{e}(S)+r\left[b-a-m_{e}(S)\right] .
$$

Since this inequality holds for arbitrarily large $k$, we must have $m_{e}(S)=0$ and hence $m\left(E_{r}{ }^{(n)}\right)=0$.

We next note that the set of points at which $D_{-}=+\infty$ is of measure zero. For, by a theorem of G. C. Young, ${ }^{*}$ except in a denumerable set the upper derivative on either side is greater than or equal to the lower derivative on the other. Hence, almost everywhere in the set of points where $D_{-}=+\infty$, we have also $D^{+}=+\infty$. But the set of points at which $D^{+}=+\infty$ and $D_{-} \neq-\infty$ is of measure zero.

Since $D^{+} f(x)=-D_{-} f(-x)$, the measure of the set of points at which either $D^{+}=-\infty$ or simultaneously $D_{-}=-\infty$ and $D^{+} \neq+\infty$ is also of measure zero. Summarizing, we can now say that, in the set of points at which either $D^{+}$or $D_{-}$is either $+\infty$ or $-\infty$, we necessarily have $D^{+}=+\infty$ and $D_{-}=-\infty$ at every point with the possible exception of a set of measure zero.

There remains to be considered the set of points at which both $D^{+}$and $D_{-}$are finite. We shall show that, almost everywhere in this set, $D^{+}=D_{-}$. By the previously mentioned theorem of $\mathrm{G}$. C. Young, $D^{+} \geqq D_{-}$except in a denumerable set. Accordingly, we now let $E$ denote the set of points at which $D^{+}>D_{-}$, both being finite. Classifying, we have

$$
E=\sum_{r_{1}, r_{2}, r_{8}, r_{4}} E_{r_{1}, r_{2}, r_{8}, r_{4}}
$$

* It is not necessary to refer to this theorem if we wish to use the Vitali Theorem again to show that the set of points at which, for example, $D_{-}>D^{+}$is of measure zero. However, the denumerability of this set can be seen in an elementary way as follows. Denote the set of points at which $D_{-}>D^{+}$by $E$. Then $E=\sum_{n, r} E_{r}{ }^{(n)}$, where $E_{r}{ }^{(n)}$ is the set of points $x$ of $E$ such that for every $x^{\prime}$ between $x-1 / n$ and $x$ and for every $x^{\prime \prime}$ between $x$ and $x+1 / n$, we have

$$
\frac{f(x)-f\left(x^{\prime}\right)}{x-x^{\prime}}>r>\frac{f\left(x^{\prime \prime}\right)-f(x)}{x^{\prime \prime}-x},
$$

and where the summation is over all pairs $(n, r), n$ being a positive integer and $r$ being rational. Since two points of $E_{r}^{(n)}$ are at least at distance $1 / n$ apart, $E_{r}^{(n)}$, and therefore $E$, is denumerable. 
where $E_{r_{1}, r_{2}, r_{3}, r_{4}}$ denotes the set of points where $r_{1}>D^{+}>r_{2}>r_{3}>$ $D_{-}>r_{4}$ and the summation is over all quadruples of rational numbers $r_{1}, r_{2}, r_{3}, r_{4}$ such that $r_{1}>r_{2}>r_{3}>r_{4}$. Moreover,

$$
E_{r_{1}, r_{2}, r_{8}, r_{4}}=\sum_{n=1}^{\infty} E_{r_{1}, r_{2}, r_{8}, r_{4}}^{(n)}
$$

where $E_{r_{1}, r_{2}, r_{3}, r_{4}}^{(n)}$ denotes the set of points $x$ of $E_{r_{1}, r_{2}, r_{3}, r_{4}}$ such that, for every $x^{\prime}$ between $x-1 / n$ and $x$, we have $f(x)-f\left(x^{\prime}\right)$ $>r_{4}\left(x-x^{\prime}\right)$ and, for every $x^{\prime}$ between $x$ and $x+1 / n$, we have $f\left(x^{\prime}\right)-f(x)<r_{1}\left(x^{\prime}-x\right)$. It will suffice to show that $m\left(E_{r_{1}, r_{2}, r_{3}, r_{4}}^{(n)}\right)$ $=0$. Let $(a, b)$ be any interval of length less than $1 / n$ with end points in $E_{r_{1}, r_{2}, r_{3}, r_{4}}^{(n)}$ and let $S$ be the set of points of $E_{r_{1}, r_{2}, r_{3}, r_{1}}^{(n)}$ interior to $(a, b)$. We may substitute $r_{2}$ and $r_{4}$, respectively, for $k$ and $r$ in the inequality obtained above, and thus have

$$
f(b)-f(a) \geqq r_{2} m_{e}(S)+r_{4}\left[b-a-m_{e}(S)\right] .
$$

A similar reasoning gives

$$
f(b)-f(a) \leqq r_{3} m_{e}(S)+r_{1}\left[b-a-m_{e}(S)\right],
$$

and it results that

$$
\frac{m_{e}(S)}{b-a} \leqq \frac{r_{1}-r_{4}}{r_{1}-r_{4}+r_{2}-r_{3}}
$$

The right hand member of this inequality being a constant less than 1 , it follows that $E_{r_{1}}{ }^{(n)} r_{2}, r_{3}, r_{4}$ can have exterior metric density 1 at no point. Hence $m\left(E_{r_{1}, r_{2}, r_{3}, r_{4}}^{(n)}\right)=0$. This completes the proof of the theorem.

Although in the proof we have tacitly assumed $f(x)$ to be defined on an interval, the method of proof is independent of this assumption and $f(x)$ may be regarded as defined on an arbitrary point set.

JACKSON, OHIO 